\title{
Congenital skull deformity of the right parietal-occipital area in a term neonate: A case report
}

\author{
Theodora Boutsikou ${ }^{1, \text { N*}^{*}}$, Adamantia Krepi ${ }^{1, \#}$, Zoi Iliodromiti ${ }^{1}$, Eleni Karapati ${ }^{1}$, Rozeta Sokou ${ }^{1}$ and Nicoletta Iacovidou $^{1}$ \\ ${ }^{1}$ Neonatal Department, Aretaieio Hospital, National and Kapodistrian University of Athens, Greece \\ \#T. Boutsikou and A. Krepi should be considered joint first author
}

\begin{abstract}
Congenital depression of the neonatal skull is a rare entity. Most of the cases have been attributed to obstetric trauma, mainly due to use of forceps, while the minority of cases occurs from intrauterine moulding of the fetal skull due to pressure against maternal bone structures. In the majority of uncomplicated depressions there is gradual resolution, although surgical intervention has also been reported in the literature. We present the case of a term infant born by cesarean section presenting with a skull deformity of the right parietal- occipital area with no accompanying fracture that gradually resolved within 18 months with no neurologic sequelae.
\end{abstract}

\section{Introduction}

Congenital depression of the neonatal skull is a well-defined but rare entity with an incidence of $0.01 \%$ [1]. Most of the cases have been attributed to obstetric trauma, mainly due to use of forceps or manoeuvres during a complicated delivery. The minority of the cases occurs from intrauterine moulding of the fetal skull due to pressure against maternal bones or uterine myomas. Methods of treatment depend on the cause, history of obstetric trauma and the possibility of neurologic deficit, ranging from conservative management to surgical intervention. In the majority of uncomplicated depressions there is gradual resolution.

\section{Case presentation}

We report a case of a term, female infant, first child of nonconsanguineous and healthy parents, born by caesarean section due to lack of progress in labor. The newborn's transition was uneventful and APGAR scores were 9 and 10 at $1^{\text {st }}$ and $5^{\text {th }}$ minute of life, respectively. Birth weight was $3670 \mathrm{~g}$, length and head circumference (HC) were 52 and $35.5 \mathrm{~cm}$, respectively. On the first physical examination, a deformity of the right parietal-occipital area of the skull was noticed, giving the impression that it had been pressed against mother's pelvis (Figures 1 and 2). Otherwise, physical examination, including neurological status, was normal. Skull F+P x-rays were negative for fracture (Figure 3) and a brain ultrasound did not demonstrate any underlying hematoma, midline shift or hemorrhage. Craniofacial consultation suggested pressure against maternal pelvis as the causative factor of the deformity, no further investigation was required and a follow-up appointment was scheduled at 3 months of age.

On the follow-up examination, the infant had normal developmental milestones, her neurological examination was unremarkable and the head circumference was within the appropriate range for her age (HC: $39 \mathrm{~cm}$ ). The skull deformity though, remained the same and repeated skull $x$-ray was again normal.

At the age of 18 months no deformity could be detected, and neurologic development of the infant was normal for her age.

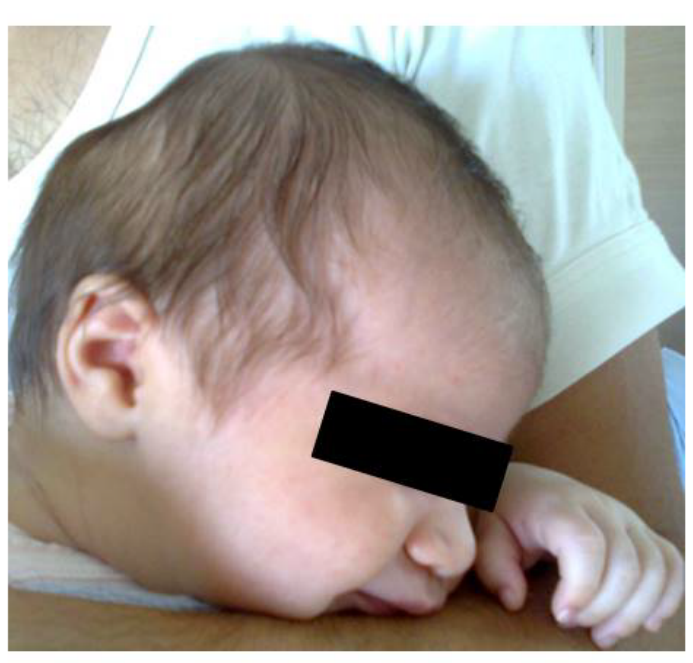

Figure 1. Skull deformity of the right parietal-occipital area

\section{Discussion}

Congenital depression of the neonatal skull has an incidence of $0.01 \%(1 / 10,000)$ in Western countries [1,2]. These deformities are usually caused by exaggerated or prolonged pressure applied on fetal skull in utero or during delivery and presence of fracture is not always the case [2]. A bony depression of more than $5 \mathrm{~mm}$ may impinge on

*Correspondence to: $\mathrm{T}$. Boutsikou, MD, PhD, Neonatal Department, Medical School, National and Kapodistrian University of Athens, 76 Vas. Sophias Avenue, GR-11528, Athens, Greece, E-mail: theobtsk@gmail.com

Key words: congenital skull depression, fracture, neonate, obstetric trauma

Received: October 12, 2020; Accepted: October 22, 2020; Published: October 25,2020 


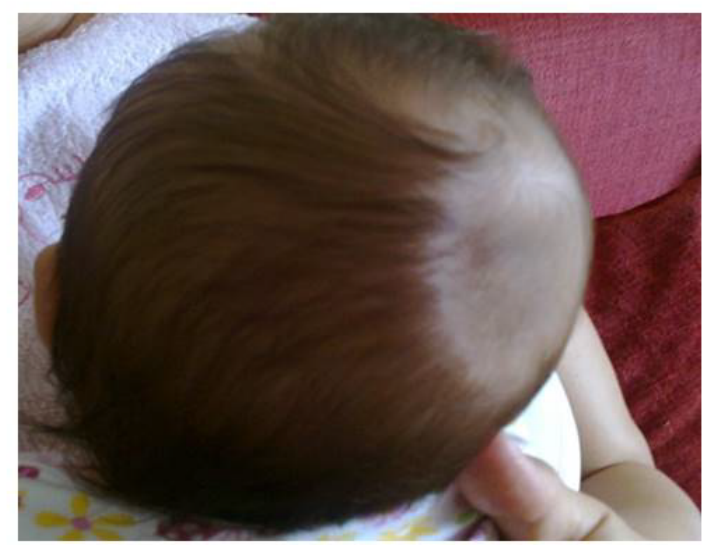

Figure 2. Skull deformity of the right parietal-occipital area

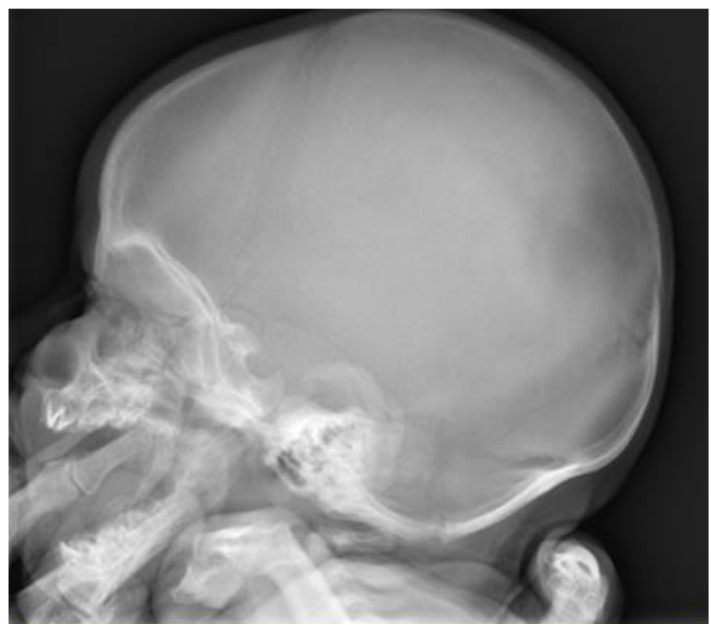

Figure 3. Skull x-ray of the neonate

the cerebral cortex, resulting in compression of the brain, leading to cerebral edema and decreased blood flow $[2,3]$. The skull may be pressed against maternal structures in utero (such as L5, vertebrae, sacral promontory, symphysis pubis, ischial spine, asymmetrical or contracted pelvis, exostosis of lumbar vertebrae, fracture of pelvis) $[2,3]$; furthermore pressure may be exerted by fetal arm, wrist, fingers or foot [2]. In our case no leiomyomas or fibromas of the uterus were detected, which could also act as causative factors. However, the most common mechanism of skull depression still remains the obstetric trauma during delivery via the use of forceps or vacuum [2] - none of which were used during delivery of our newborn. As mentioned in the beginning, skull deformities due to idiopathic reasons are rare [1], and can be defined as such in cases where no causative factor can be identified. The majority of spontaneous cases have a very good prognosis.

In the case of a deformed skull depression, skull $\mathrm{x}$-ray is essential for exclusion of fracture. Neurosurgical intervention is the first-line treatment in case of fracture, but recently there has been good evidence of spontaneous elevation and remarkable outcomes from non-surgical management. To date, both types (deformation without fracture or with fracture) are primarily treated conservatively, as they tend to heal themselves within four months [1]. The surgical options include open cranioplasty and percutaneous microscrew elevation [4]. Mandatory urgent surgery is indicated for cases of present cerebrospinal fluid leak, detection of a foreign body or when evacuation of a hematoma is necessary [4]. Non-surgical methods providing a favorable cosmetic outcome include elevation of the depressed area with negative pressure devices such as vacuum extractor or breast pump [2], combined with the avoidance of any postoperative complications (surgical scarring, wound infection). The conservative approach also favors breastfeeding avoiding separation of mother-infant. Successful treatment using a vacuum pump was first described in the 1970s by Schrager [5]. Prior to this, a CT scan has to be performed in order to exclude intracranial hemorrhage and repeated likewise after the procedure.

\section{Conclusion}

We presented a case of a term infant born by cesarean section presenting with a skull deformity of the right parietal- occipital area with no accompanying fracture that gradually resolved within 18 months with no neurologic sequelae. In our case the medical and perinatal history of the parturient was not associated with the respective malformation, since there was no obstetric trauma reported. The infant was managed conservatively with no neurological complications.

Non-surgical approach to congenital skull depression should be preferred before neurosurgical treatment $[2,6]$, for its effectiveness and safety concerning the risk of postoperative complications. However, each case should be guided by the severity of the fracture, if any, and possible underlying intracerebral injury [3].

The lack of specific guidelines in the literature could possibly lead to over-estimation and aggressive treatment, so neonatologists and obstetricians should be familiar with this clinical entity.

\section{References}

1. Hanlon L, Hogan B, Corcoran D, Ryan S (2006) Congenital depression of the neonatal skull: a self-limiting condition. Arch Dis Child Fetal Neonatal Ed 91: F272. [Crossref]

2. Ben-Ari Y, Merlob P, Hirsch M, Reisner SH (1986) Congenital depression of the neonatal skull. Eur J Obstet Gynecol Reprod Biol 22: 249-255. [Crossref]

3. Preston D, Jackson S, Gandhi S (2015) Non-traumatic depressed skull fracture in a neonate or 'ping pong' fracture. BMJ Case Rep 18: bcr2014207077. [Crossref]

4. Ballestero MF, De Oliveira RS (2019) Closed depressed skull fracture in childhood reduced with suction cup vacuum method: Case report and a systematic literature review. Cureus 11: e5205 [Crossref]

5. Schrager GO (1970) Elevation of depressed skull fracture with a breast pump. J Pediatr 77: 300-301. [Crossref]

6. Raynor R, Parsa M (1968) Nonsurgical elevation of depressed skull fracture in an infant. J Pediatr 72: 262-264. [Crossref]

Copyright: (C2020 Boutsikou T. This is an open-access article distributed under the terms of the Creative Commons Attribution License, which permits unrestricted use, distribution, and reproduction in any medium, provided the original author and source are credited. 